\title{
Pyrolysis behavior and micromorphology investigation on aluminum based solid-liquid compound fuel
}

\author{
Xinghua Liu ${ }^{1, a}$, Hua Yan ${ }^{1, b}$, Yongfeng Luo ${ }^{1, c}$, Daxi Wang ${ }^{1, d}$, Shuyuan $\mathrm{Li}^{1, \mathrm{e}, *}$, \\ Yue $\mathrm{Ma}^{1, \mathrm{f}}$, Xiaoyan Liu ${ }^{1, g}$ and Yi Zhao ${ }^{1, h}$ \\ ${ }^{1}$ China University of petroleum-Beijing, Beijing 102249, China; \\ alxhlxh052@126.com, 'bhlary@163.com, cyfluo@163.com, dwdxi@cup.edu.cn, esyli@cup.edu.cn,

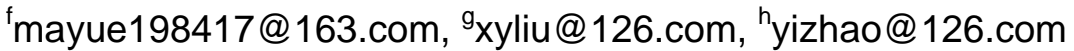

Keywords: Thermal decomposition, micromorphology, compound fuel.

\begin{abstract}
The compatibility, stability and the pyrolysis behavior of aluminum based solid-liquid compound fuel were systematically investigated by thermogravimetric analysis (TGA). The compound fuel lost its $30 \%$ weight before the temperature rose to $150^{\circ} \mathrm{C}$. AP decomposed at $210^{\circ} \mathrm{C}$ and lead to $50 \%$ weight loss. The reaction between aluminum and oxygen was accelerated at $6600^{\circ} \mathrm{C}$ since the melting of solid combustible agent. The total weight loss after $1000^{\circ} \mathrm{C}$ pyrolysis is about $80 \%$ for nitrogen atmosphere. On the contrary, the total weight loss for oxygen atmosphere is approximate $70 \%$ because of the generation of aluminum oxide. The microscopic structures and the elementary composition of the pyrolysis products were analyzed using scanning electron microscopy-energy dispersive spectroscopy (SEM-EDS). The structures of the products after $200^{\circ} \mathrm{C}$ pyrolysis are spherical and flaky. All these structures are destroyed after $1000^{\circ} \mathrm{C}$ pyrolysis. Instead, massive structures were found in the products. Aluminum is the main element in the final pyrolysis products for all conditions. The second-most element in the product for nitrogen atmosphere is carbon. Carbon deposited accompany with the decomposition of paraffin or other organic components. The second-most element in the product for oxygen atmosphere is oxygen. This conclusion is attributed to the formation of aluminum oxide.
\end{abstract}

\section{Introduction}

The fundamental investigations of solid-liquid compound fuel have attracted great interests since the Vietnam War in 1970s for the outstanding lethality of fuel air explosives (FAE) weapons. Solid-liquid compound fuel is the basis for the fuel air explosives FAE. The studies on the compatibility among different components and the explosion mechanism of solid-liquid compound fuel are urgent to be solved. In 1977, R. Knystautas [1] found that the explosive mechanism of FAE is on the basis of SWACER, which is the abbreviation of Shock Wave Amplification by Coherent Energy Release. A. A. Borisov [2] reported that rapid detonation and reaction shock wave can also generate powerful damage effect, not just restricted to explosive vapor clouds. Domestic investigations have been lasted for twenty years and mainly focus on the properties of different components and the details of reaction process [3-5]. The researches on denotation mode by photochemical initiation and catalytic chemical initiation have also been reported [6].

Solid-liquid aluminum based compound fuel is a explosive mixture, which is composed of solid combustible agent (aluminum powder), liquid combustible agent, oxidant, sensibilizer, passivator and adhesive. The premise of a excellent compound fuel is good compatibility, which means different components do not react within the specific conditions. In the present study, the inner compatibility, stability and the pyrolysis behavior of aluminum based solid-liquid compound fuel was investigated by TGA. The microscopic structures and the elementary composition of the pyrolysis products were analyzed using SEM-EDS. 


\section{Experimental}

\section{Preparation of solid-liquid aluminum based compound fuel.}

Raw materials. Solid combustible agent: spherical and flaky aluminum powder (99.99\%), Hunan Jintian aluminum Industry Co. Ltd.; Liquid combustible agent: aviation kerosene, Qingdao Sanho Envirionmental Protection Technology Co. Ltd.; Oxidant: AP (AR), Tianjing Chemical Reagent Factory; Sensibilizer: RDX (AR), Tianjing Chemical Reagent Factory; passivator: paraffin, Shanghai Joule wax Co. Ltd. All the raw materials were blended using homomixer at a proper speed and order. The obtained compound fuel was kept in an aluminum can.

\section{TGA and SEM-EDS tests}

The TGA test was conducted by Microcomputer Differential Thermal Balance (HCT-3, Beijing Henven Scientific Instrument Factory). Test conditions: heating rate is $10 \sim 30^{\circ} \mathrm{C} / \mathrm{min}$, the mass of sample is about $15 \mathrm{mg}, \mathrm{N}_{2} / \mathrm{O}_{2}$ atmosphere, flow rate is $60 \mathrm{ml} / \mathrm{min}$, maximum temperature is $1000^{\circ} \mathrm{C}$.

The SEM-EDS test was performed by Cold Field Emission Scanning Electron Microscope (SU8010, Hatichi). The microstructures of the pyrolysis product obtained from different temperature $\left(450{ }^{\circ} \mathrm{C}, 1000{ }^{\circ} \mathrm{C}\right)$ were investigated and the elemental composition of the sample surface was determined in order to understand the pyrolysis mechanism.

\section{Results and Discussion}

\section{TGA results}

The TG curves of aluminum based solid-liquid compound fuel at nitrogen and oxygen atmosphere with different heating rate are shown in Fig. 1. From (a) in Fig. 1, the curves with different heating rate have the same trend of losing weight. There are three stages during the whole process. At the first stage (temperature is lower than $150^{\circ} \mathrm{C}$ ), the compound fuel losses its $30 \%$ weight gradually. At the second stage (temperature interval is between 150 and $200^{\circ} \mathrm{C}$ ), the weight of the compound fuel keeps constant. At the third stage (temperature is greater than 200), when the temperature reaches $210^{\circ} \mathrm{C}$, the curves fall down abruptly, and the weight loss is about $50 \%$. As the temperature goes up, the weight of compound fuel is slightly increased, which can be ignored for its small value.

As to (b) in Fig. 1, the curves with different heating rate also have the same trend of losing weight. At the first and second stages, the weight loss of compound fuel at nitrogen and oxygen atmosphere shares the same tendency. However, sensible difference can be observed when temperature is greater than $200^{\circ} \mathrm{C}$. The break point at oxygen atmosphere is as the same as that of at nitrogen atmosphere. But when the temperature is between the interval of $300-600{ }^{\circ} \mathrm{C}$, the weight of compound fuel increases slightly with the increase of temperature. When the temperature reaches $660^{\circ} \mathrm{C}$, the weight of compound fuel increases obviously with the increase of temperature.

The weight loss curves at different conditions can help to understand the compatibility, stability and pyrolysis behavior of compound fuel. At the first stage, the weigh decreases gradually because of the evaporation of liquid combustible agent. The weight decreases dramatically at $210^{\circ} \mathrm{C}$ for the pyrolysis of AP, which gives off huge amounts of gases and heat. Another notable point is $660^{\circ} \mathrm{C}$ at oxygen atmosphere, the weight increase can be attribute to the oxidation of aluminum. As is known to us all, alumina film blocks the further oxidation of aluminum. However, when temperature reaches $660^{\circ} \mathrm{C}$, the melting point of aluminum, liquid aluminum react rapidly with oxygen to give aluminum oxide. This reaction can cause the increase of compound fuel weight. In addition, some organic components, such as paraffin and adhesive, can decompose at high temperature or react with oxygen, which would decrease the weight of compound fuel. Because the content of such components are very low, so their effect is not observed from TG results. 

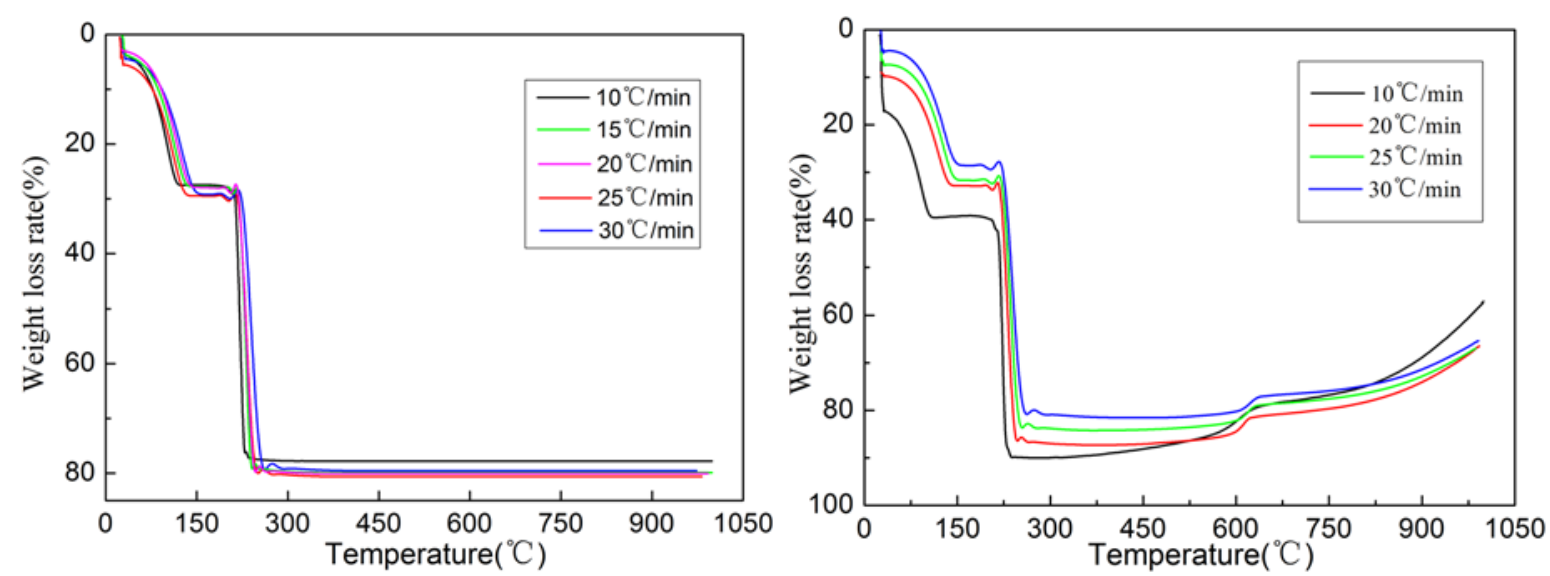

Fig. 1 TG curves of compound fuel at different heating rate. (a) Nitrogen atmosphere (b) Oxygen atmosphere

\section{Microscopic structures and elementary composition analysis}

In order to fully understand the pyrolysis mechanism and further confirmation of TG conclusion, SEM-EDS analysis of pyrolysis products at $200^{\circ} \mathrm{C}, 500^{\circ} \mathrm{C}$ and $1000^{\circ} \mathrm{C}$ were performed. The results are shown in Fig. 2. Figure 2(a) and 2(b) are the microstructure of pyrolysis product at $200^{\circ} \mathrm{C}$. The spherical and flaky aluminum are clearly showed and well blended. Figure 2(c) and 2(d) are the microstructure of pyrolysis product at $500^{\circ} \mathrm{C}$. A few pore structures can be found, which indicates gaseous product has been released. This result is consistence with the TG analysis: AP decompose at $210^{\circ} \mathrm{C}$. Figure 2(e) and 2(f) are the microstructure of pyrolysis product at $1000^{\circ} \mathrm{C}$. The spherical or flaky structures of aluminum are partially destroyed in Fig. 2(e), while in Fig. 2(f), such structures are totally disappeared, and massive structure can be observed.

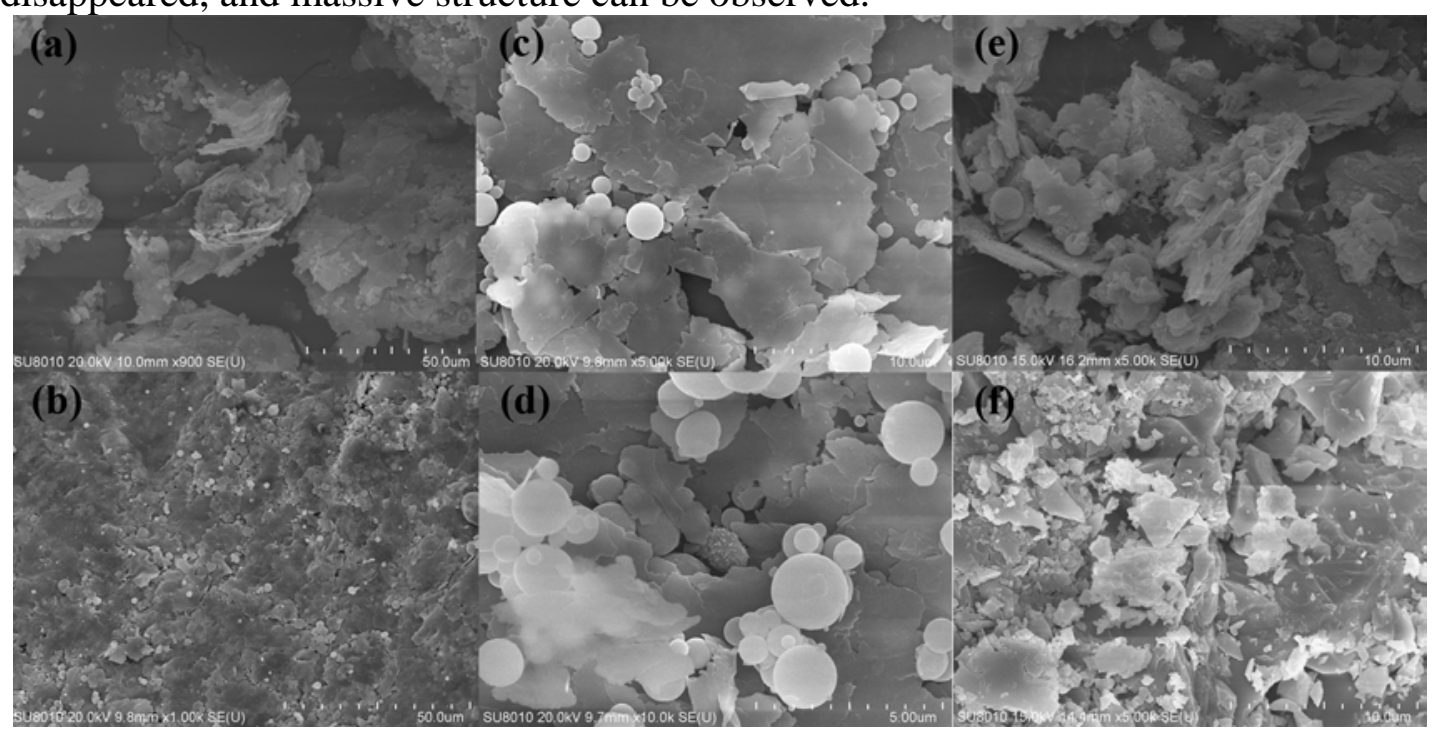

Fig. 2 Microstructures of pyrolysis products at (a)Nitrogen, $200^{\circ} \mathrm{C}$; (b) Oxygen, $200^{\circ} \mathrm{C}$; (c)Nitrogen, $500^{\circ} \mathrm{C}$; (d) Oxygen, $500^{\circ} \mathrm{C}$; (e)Nitrogen, $1000^{\circ} \mathrm{C}$; (f) Oxygen, $1000^{\circ} \mathrm{C}$.

Table 1 Elementary composition of pyrolysis products at various conditions.

\begin{tabular}{|c|c|c|c|c|c|}
\hline $\begin{array}{c}\text { Pyrolysis } \\
\text { product }\end{array}$ & Atmosphere & $\mathrm{C}(\mathrm{wt} \%)$ & $\mathrm{N}(\mathrm{wt} \%)$ & $\mathrm{O}(\mathrm{wt} \%)$ & $\mathrm{Al}(\mathrm{wt} \%)$ \\
\hline \multirow{2}{*}{$200^{\circ} \mathrm{C}$} & $\mathrm{N}_{2}$ & 16.23 & 9.32 & 14.32 & 60.13 \\
\cline { 2 - 6 } & $\mathrm{O}_{2}$ & 22.49 & 10.43 & 14.68 & 52.4 \\
\hline \multirow{2}{*}{$500^{\circ} \mathrm{C}$} & $\mathrm{N}_{2}$ & 15.4 & - & 5.4 & 79.2 \\
\cline { 2 - 6 } & $\mathrm{O}_{2}$ & 14.35 & - & 8.37 & 79.28 \\
\hline \multirow{2}{*}{$1000^{\circ} \mathrm{C}$} & $\mathrm{N}_{2}$ & 13.99 & - & 9.64 & 76.38 \\
\cline { 2 - 6 } & $\mathrm{O}_{2}$ & 8.35 & - & 41.28 & 50.37 \\
\hline
\end{tabular}


EDS results are summarized in Table 1 . The main elements of compound fuel are carbon, nitrogen, oxygen and aluminum. In the product of $200{ }^{\circ} \mathrm{C}$, Al element accounts for about $60 \% \mathrm{C}, \mathrm{N}$ and $\mathrm{O}$ elements are approximately equal. In the product of $500^{\circ} \mathrm{C}$, the content of $\mathrm{C}$ remained about the same, while $\mathrm{O}$ content decreases. In addition, $\mathrm{N}$ has not detected neither in nitrogen nor in oxygen atmosphere. In the product of $1000^{\circ} \mathrm{C}$, half of the $\mathrm{C}$ content has been lost at oxygen atmosphere comparing to the $200{ }^{\circ} \mathrm{C}$ and $500^{\circ} \mathrm{C}$. The result can be explained by the combustion of organic components. The $\mathrm{O}$ content at oxygen atmosphere is four times as much as that of at nitrogen atmosphere. Obviously, the high content of $\mathrm{O}$ at oxygen atmosphere can be due to the formation of aluminum oxide.

\section{Conclusion}

The aluminum based solid-liquid compound fuel was prepared successfully. Heating rate has little effect on the pyrolysis behavior of compound fuel. According to the TGA results, the inner compatibility of the compound fuel at low temperature is good, while the liquid combustible agent evaporates as the temperature increase. The end point of liquid combustible agent evaporation is about $150^{\circ} \mathrm{C}$ and the total weight loss at this stage is about $30 \%$. The first break point locates at $210^{\circ} \mathrm{C}$, about $50 \%$ weight is lost for the decomposition of AP at this temperature. The components containing $\mathrm{N}$ element decompose completely when the temperature is $500^{\circ} \mathrm{C}$, which has been confirmed by EDS results. When temperature reaches $660^{\circ} \mathrm{C}$, the alumina film on the surface of aluminum is destroyed for the phase transformation of aluminum. The reaction between aluminum and oxygen is accelerated and leads to the increase of sample weight. The spherical or flaky structures of solid combustible agent were disappeared, while the massive structure of the product can be observed after $1000^{\circ} \mathrm{C}$ pyrolysis.

\section{References}

[1] Knystautas R, Lee J H. Mechanisms of initiation of detonation in explosive vaper clouds. AFOSR-TR-78-0456, 1978.

[2] Borisov A. A. Fuel-air Explosives. Final Report: Institute of Chemical Physics, Russian Academy of Sciences, 1994.

[3] Jingming Pei, Xuezhong Xu. Properties of anti-high-loading Fuel Air Explosive. Chinese Journal of Explosives \& Propellants.. Vol. 28 (2005), p. 31-34.

[4] Xuezhong Xu, Jingming Pei. Dispersion characteristics of single-event FAE. Chinese Journal of Explosives \& Propellants. Vol. 1 (2000), p. 47-49.

[5] Xuezhong Xu, Weihai Ying. The thermal radication of fuel-air mixtures with single-event ignition. Initiators \& Pyrotechnics. Vol. 4 (1999), p. 14-17.

[6] Xuezhong $\mathrm{Xu}$, Mingjing Pei. Explosive reaction characteristics on hydrocarbon and fluorine agent. Chinese Journal of Explosives \& Propellants. Vol. 3 (1999), p. 36-38. 